\title{
TIME-FRACTIONAL HEAT CONDUCTION IN A FINITE COMPOSITE CYLINDER WITH HEAT SOURCE
}

\author{
Stanistaw Kukla, Urszula Siedlecka \\ Department of Mathematics, Czestochowa University of Technology \\ Czestochowa, Poland \\ stanislaw.kukla@im.pcz.pl,urszula.siedlecka@im.pcz.pl
}

Received: 29 March 2020; Accepted: 11 May 2020

\begin{abstract}
In this paper, the effect of the fractional order of the Caputo time-derivative occurring in heat conduction models on the temperature distribution in a finite cylinder consisting of an inner solid cylinder and an outer concentric layer is investigated. The inner cylinder (core) and the cylindrical layer are in perfect thermal contact. The Robin boundary condition on the outer surface and the Neumann conditions on the ends of the cylinder are assumed. An internal heat source is represented in the mathematical model by taking into account in the heat conduction equation of a function which depends on the space and time variable. An analytical solution of the problem is derived in the form of the double series of eigenfunctions. Numerical examples are presented.
\end{abstract}

MSC 2010: 26A33, 34A08, $80 A 20$

Keywords: fractional heat conduction, Caputo derivative, composite cylinder, heat source

\section{Introduction}

In recent years, an increasing interest in applications of fractional calculus in engineering, economical and biological sciences can be observed. It is related to this that the researchers search higher accurate description of the investigated phenomena or processes. The fractional derivatives and integrals occur in fractional differential equations which describe the modelled processes. History of the fractional calculus development is presented in the papers [1,2]. The properties of the fractional derivatives and integrals and selected methods to solving the fractional differential equations are presented for example in the books [3-5] and papers [6, 7].

Mathematical modelling of the heat conduction is the subject of many papers, see [8-24]. Applying the Fourier's law, a partial differential equation of parabolic type with time derivative of the first order is obtained. This mathematical model of heat conduction has a non-physical property, that the speed of thermal signal in the medium is infinity. In order to avoid this non-realistic property, a generalized 
Fourier law is applied. The generalization relies on introducing a phase-lag parameter [8] to the classical Fourier law. The obtained equation together with the energy equation [9] leads to a hyperbolic heat conduction equation. Replacing the time-derivatives in the generalized Fourier law and energy equation by fractional derivatives, a fractional heat conduction equation is obtained [10].

The fractional mathematical models for description of the heat transfer are used in the articles [10-24]. These articles are only a part of the numerous research papers subjected to the heat transfer which have been published in recent years. The fractional heat conduction (FHC) in slabs and plates is investigated in papers [11-14], FHC in layered spheres is the subject of the papers [15-19]. The articles [20-24] are devoted to the problems of FHC in cylinders. In the paper [20], the fractional heat conduction equation based on the time-fractional Fourier law in the orthogonal coordinate system was derived. As a special case, the FHC equation in the cylindrical coordinate system was obtained. A solution of the problem was presented for an infinite medium. The Dirichlet and two types of Neumann problems for a cylinder in the case of axial symmetry have been solved in paper [21]. The determined temperature distributions were used for calculation of the associated thermal stresses in the cylinder. The FHC and fractional thermoelasticity problems for an infinite solid cylinder were considered in the paper [22]. A fractional model of the heat conduction in an infinite long hollow cylinder is given in paper [23]. The effect of the derivative fractional order and variable thermal conductivity on temperature and stresses in the cylinder were numerically investigated. In paper [24], a solution to the FHC problem for a finite hollow cylinder under Gaussian-distributed heat flux is presented.

In this paper, a solution of the problem to the fractional heat conduction in a composite cylinder with heat sources is presented. The heat conduction is considered in radial and axial directions. The differential equation governing the heat conduction involves the Caputo time-derivative. Numerical analysis shows the effect of the derivative fractional order on the temperature distribution in the cylinder.

\section{Formulation of the problem}

Consider the time-fractional heat conduction problem for a finite cylinder consisting of an inner solid cylinder and an outer concentric layer (Fig. 1).

The differential equation in cylindrical coordinates governing the temperature $T_{i}(t, r, z)$ is given as

$$
\nabla^{2} T_{i}(t, r, z)+\frac{1}{\lambda_{i}} g_{i}(t, r, z)=\frac{1}{a_{i}} \frac{\partial^{\alpha} T_{i}}{\partial t^{\alpha}}(t, r, z), \quad z \in[0, H], r \in\left[r_{i-1}, r_{i}\right], i=1,2
$$

where $\lambda_{i}$ is the constant thermal conductivity, $a_{i}$ is the constant thermal diffusivity, $g_{i}(t, r, z)$ is the volumetric energy generation, $\alpha$ denotes the fractional order of 
the Caputo derivative with respect to time $t, r_{0}=0$ and the Laplace operator $\nabla^{2}$ is given by

$$
\nabla^{2} T_{i}=\frac{1}{r} \frac{\partial}{\partial r}\left(r \frac{\partial T_{i}}{\partial r}\right)+\frac{\partial^{2} T_{i}}{\partial z^{2}}, \quad i=1,2
$$

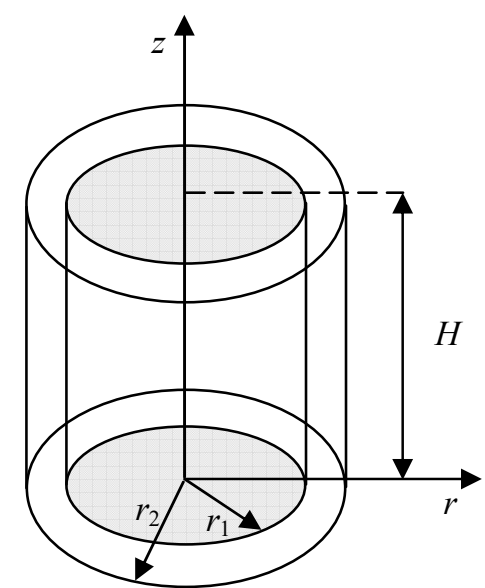

Fig. 1. A sketch of the considered finite cylinder

The Caputo derivative is defined as

$$
\frac{d^{\alpha} f(t)}{d t^{\alpha}}=\frac{1}{\Gamma(m-\alpha)} \int_{0}^{t}(t-\tau)^{m-\alpha-1} \frac{d^{m} f(\tau)}{d \tau^{m}} d \tau, \quad m-1<\alpha<m, m \in N
$$

In this paper, we consider the Caputo derivative fractional order $\alpha$ in the interval $(0,1]$.

We assume the following boundary conditions:

$$
\begin{gathered}
\left|T_{1}(t, 0, z)\right|<\infty \\
\left.\lambda_{2} \frac{\partial T_{2}}{\partial r}\right|_{r=r_{2}}=a_{\infty}\left(T_{\infty}(t)-T_{2}\left(t, r_{2}, z\right)\right) \\
\left.\frac{\partial T_{i}}{\partial z}\right|_{z=0}=0, \quad i=1,2 \\
\left.\frac{\partial T_{i}}{\partial z}\right|_{z=H}=0, \quad i=1,2
\end{gathered}
$$


and the conditions of perfect thermal contact at interface $\left(r=r_{1}\right)$

$$
\begin{aligned}
& T_{1}\left(t, r_{1}, z\right)=T_{2}\left(t, r_{1}, z\right) \\
& \left.\lambda_{1} \frac{\partial T_{1}}{\partial r}\right|_{r=r_{1}}=\left.\lambda_{2} \frac{\partial T_{2}}{\partial r}\right|_{r=r_{1}}
\end{aligned}
$$

where $a_{\infty}$ is the heat transfer coefficient and $T_{\infty}$ is the ambient temperature. Moreover, we assume the initial condition

$$
T_{i}(0, r, z)=F_{i}(r, z), \quad i=1,2
$$

We search for a solution to the problem (1), (4-10), in the form

$$
T_{i}(t, r, z)=T_{\infty}(t)+\psi_{i}(t, r, z), \quad i=1,2
$$

where functions $\psi_{i}$ satisfy the fractional differential equation

$$
\nabla^{2} \psi_{i}(t, r, z)=\frac{1}{a_{i}} \frac{\partial^{\alpha} \psi_{i}(t, r, z)}{\partial t^{\alpha}}+\frac{1}{a_{i}} \frac{\partial^{\alpha} T_{\infty}(t)}{\partial t^{\alpha}}-\frac{1}{\lambda_{i}} g_{i}(t, r, z), \quad i=1,2
$$

and the following boundary and initial conditions:

$$
\begin{gathered}
\left|\psi_{1}(t, 0, z)\right|<\infty \\
\psi_{1}\left(t, r_{1}, z\right)=\psi_{2}\left(t, r_{1}, z\right) \\
\left.\lambda_{1} \frac{\partial \psi_{1}}{\partial r}\right|_{r=r_{1}}=\left.\lambda_{2} \frac{\partial \psi_{2}}{\partial r}\right|_{r=r_{1}} \\
\left.\lambda_{2} \frac{\partial \psi_{2}}{\partial r}\right|_{r=r_{2}}=-a_{\infty} \psi_{2}\left(t, r_{2}, z\right) \\
\left.\frac{\partial \psi_{i}}{\partial z}\right|_{z=0}=0, \quad i=1,2 \\
\left.\frac{\partial \psi_{i}}{\partial z}\right|_{z=H}=0, \quad i=1,2 \\
\psi_{i}(0, r, z)=F_{i}(r, z)-T_{\infty}(0)
\end{gathered}
$$


We search for a solution to the problem (12)-(19) by using the separation of variables method, in the form of a double series

$$
\psi_{i}(t, r, z)=\theta(t) R_{i}(r) Z(z), \quad i=1,2
$$

The functions $Z$ and $R$ are obtained as solutions of two homogeneous differential equations

$$
\begin{gathered}
\frac{d^{2} Z}{d z^{2}}+\mu^{2} Z=0 \\
\frac{1}{r} \frac{d}{d r}\left(r \frac{d R_{i}}{d r}\right)+v_{i}^{2} R_{i}=0
\end{gathered}
$$

where $\mu$ and $v_{i}$ are separation constants. These functions satisfy the homogeneous boundary conditions which follow from the conditions (13)-(18)

$$
\begin{gathered}
\left.\frac{d Z}{d z}\right|_{z=0}=0,\left.\frac{d Z}{d z}\right|_{z=H}=0 \\
\left|R_{1}(0)\right|<\infty, R_{1}\left(r_{1}\right)=R_{2}\left(r_{1}\right),\left.\lambda_{1} \frac{d R_{1}}{d r}\right|_{r=\eta_{1}}=\left.\lambda_{2} \frac{d R_{2}}{d r}\right|_{r=\eta_{1}},\left.\lambda_{2} \frac{d R_{2}}{d r}\right|_{r=r_{2}}=-a_{\infty} R_{2}\left(r_{2}\right)
\end{gathered}
$$

The solutions of the eigenvalue problem (21), (23) are functions

$$
Z_{m}(z)=\cos \mu_{m} z
$$

where $\mu_{m}=\frac{m \pi}{H}, m=0,1,2, \ldots$. While the solutions of the eigenvalue problem (22), (24) are given as

$$
R_{i, m}(r)=A_{i, m} j_{0}\left(v_{i, m} r\right)+B_{i, m} y_{0}\left(v_{i, m} r\right), \quad i=1,2
$$

where

$$
\left[j_{0}\left(v_{i, m} r\right), y_{0}\left(v_{i, m} r\right)\right]= \begin{cases}{\left[J_{0}\left(v_{i, m} r\right), Y_{0}\left(v_{i, m} r\right)\right]} & \text { for } \quad \gamma_{m}>\mu_{m} \sqrt{a_{i}} \\ {[1, \ln r]} & \text { for } \quad \gamma_{m}=\mu_{m} \sqrt{a_{i}} \\ {\left[I_{0}\left(v_{i, m} r\right), K_{0}\left(v_{i, m} r\right)\right]} & \text { for } \quad \gamma_{m}<\mu_{m} \sqrt{a_{i}}\end{cases}
$$

and $v_{i, m}=\sqrt{\left|\frac{\gamma_{m}^{2}}{a_{i}}-\mu_{m}^{2}\right|}$. The functions $J_{0}, J_{1}$ and $Y_{0}, Y_{1}$ are the Bessel functions of the first and second kind, the functions $I_{0}, I_{1}$ and $K_{0}, K_{1}$ are the modified Bessel 
functions of the first and second kind, respectively. Using condition (24a), we receive that $B_{1, m}=0$ and on the basis of the homogeneous conditions (24b-d), we obtain an eigenvalue equation. The eigenvalue equation for the case of $\gamma_{m}>\mu_{m} \sqrt{a_{i}}$ is as follows:

$$
\begin{aligned}
& \left(-\lambda_{1} v_{1, m} J_{0}\left(r_{1} v_{2, m}\right) J_{1}\left(r_{1} v_{1, m}\right)+\lambda_{2} v_{2, m} J_{0}\left(r_{1} v_{1, m}\right) J_{1}\left(r_{1} v_{2, m}\right)\right) . \\
& \left(a_{\infty} Y_{0}\left(r_{2} v_{2, m}\right)-\lambda_{2} v_{2, m} Y_{1}\left(r_{2} v_{2, m}\right)\right)-\left(a_{\infty} J_{0}\left(r_{2} v_{2, m}\right)-\lambda_{2} v_{2, m} J_{1}\left(r_{2} v_{2, m}\right)\right) . \\
& \left(-\lambda_{1} v_{1, m} J_{1}\left(r_{1} v_{1, m}\right) Y_{0}\left(r_{1} v_{2, m}\right)+\lambda_{2} v_{2, m} J_{0}\left(r_{1} v_{1, m}\right) Y_{1}\left(r_{1} v_{2, m}\right)\right)=0
\end{aligned}
$$

Solving this equation for $m=0,1,2, \ldots$, we obtain sequences of roots $\gamma_{m, n}, n=1$, $2, \ldots$. The coefficients $A_{1, m}, A_{2, m}, B_{2, m}$ occurring in equation (26) are determined using conditions (24b-d):

$$
\begin{gathered}
A_{1, m}=1 \\
A_{2, m}=\frac{\pi}{2} \frac{r_{1}}{\lambda_{2}}\left(\lambda_{1} v_{1, m} J_{1}\left(r_{1} v_{1, m}\right) Y_{0}\left(r_{1} v_{2, m}\right)-\lambda_{2} v_{2, m} J_{0}\left(r_{1} v_{1, m}\right) Y_{1}\left(r_{1} v_{2, m}\right)\right) \\
B_{2, m}=-\frac{\pi}{2} \frac{r_{1}}{\lambda_{2}}\left(\lambda_{1} v_{1, m} J_{0}\left(r_{1} v_{2, m}\right) J_{1}\left(r_{1} v_{1, m}\right)-\lambda_{2} v_{2, m} J_{0}\left(r_{1} v_{1, m}\right) J_{1}\left(r_{1} v_{2, m}\right)\right)
\end{gathered}
$$

Similarly, we obtain the eigenequation and coefficients $A_{1, m}, A_{2, m}, B_{2, m}$ for $\gamma_{m}<\mu_{m} \sqrt{a_{i}}$. In this case, the equation can be obtained by replacing the Bessel functions $J_{0}, J_{1}, Y_{0}$ and $Y_{1}$ in equation (28) into the modified Bessel functions $I_{0},-I_{1}, K_{0}$ and $K_{1}$, respectively.

The functions $R_{i, m, n}$ given by equation (26) create an orthogonal set of functions, i.e. the following condition holds

$$
\frac{\lambda_{1}}{a_{1}} \int_{0}^{r_{1}} r R_{1, m, n} R_{1, m, n^{\prime}} d r+\frac{\lambda_{2}}{a_{2}} \int_{n_{1}}^{b} r R_{2, m, n} R_{2, m, n^{\prime}} d r= \begin{cases}0 & n^{\prime}=n \\ N_{m, n}^{r} & n^{\prime} \neq n\end{cases}
$$

where $N_{m, n}^{r}=\left(\frac{\lambda_{1}}{a_{1}} \int_{0}^{\eta} r R_{1, m, n}^{2}(r) d r+\frac{\lambda_{2}}{a_{2}} \int_{n}^{b} r R_{2, m, n}^{2}(r) d r\right)$. Substituting the series (20) into the differential equation (12) and using the orthogonality condition (32), we obtain the following fractional differential equation

$$
\frac{d^{\alpha} \theta_{m, n}(t)}{d t^{\alpha}}+\gamma_{m, n}^{2} \theta_{m, n}(t)=f_{m, n}(t)
$$


where

$$
\begin{aligned}
& f_{m, n}(t)=\frac{1}{N_{m, n}^{r} N_{m}^{z}}\left(\int_{0}^{H} Z_{m}(z) \int_{0}^{\eta} g_{1}(t, r, z) r R_{1, m, n}(r) d r d z+\int_{0}^{H} Z_{m}(z) \int_{n}^{b} g_{2}(t, r, z) r R_{2, m, n}(r) d r d z\right) \\
& -\frac{1}{N_{m, n}^{r} N_{m}^{z}} \frac{d^{\alpha} T_{\infty}(t)}{d t^{\alpha}} \int_{0}^{H} Z_{m}(z) d z\left(\frac{\lambda_{1}}{a_{1}} \int_{0}^{n} r R_{1, m, n}(r) d r+\frac{\lambda_{2}}{a_{2}} \int_{r_{1}}^{b} r R_{2, m, n}(r) d r\right)
\end{aligned}
$$

whereas $N_{0}^{z}=H$ and $N_{m}^{z}=\frac{H}{2}$ for $\mathrm{m}=1,2, \ldots$. Equation (33) is complemented by the initial condition which is obtained on the basis of equations (19)-(20) and the orthogonality condition (32)

$$
\theta_{m, n}(0)=\frac{1}{N_{m, n}^{r} N_{m}^{z}} \cdot\left(M_{1, m, n}+M_{2, m, n}\right)
$$

where $M_{i, m, n}=\frac{\lambda_{i}}{a_{i}} \int_{0}^{H} Z_{m}(z) \int_{r_{i-1}}^{r_{i}}\left(F_{i}(r, z)-T_{\infty}(0)\right) r R_{i, m, n}(r) d r d z, i=1,2$.

The solution of initial-value problem (33)-(34) can be presented as

$$
\begin{aligned}
\theta_{m, n}(t) & =\int_{0}^{t}(t-\tau)^{\alpha-1} E_{\alpha, \alpha}\left(-\gamma_{m, n}^{2}(t-\tau)^{\alpha}\right) f_{m, n}(\tau) d \tau \\
& +\frac{1}{N_{m, n}^{r} N_{m}^{z}} E_{\alpha}\left(-\gamma_{m, n}^{2} t^{\alpha}\right)\left(M_{1, m, n}+M_{2, m, n}\right)
\end{aligned}
$$

Finally, the solution of the initial-boundary value problem (1) and (4)-(10) is given by equation (11) where the function $\psi_{i}(t, r, z)$ is the double sum

$$
\psi_{i}(t, r, z)=\sum_{m=0}^{\infty} \sum_{n=1}^{\infty} \theta_{m, n}(t) R_{i, m, n}(r) Z_{m}(z), \quad i=1,2
$$

whereas the functions $R_{i, m, n}(r), Z_{m}(z), \theta_{m, n}(t)$ are defined by (26), (25) and (35), respectively.

\section{Numerical examples}

The analytical solution of the problem of the time-fractional heat conduction presented in the previous section will be used in numerical calculations of the temperature distribution in the finite cylinders. The computations were performed for 
the fractional models characterized by a different order of the Caputo derivatives. We assume the following geometrical and physical data: $r_{1}=0.4 \mathrm{~m}, r_{2}=0.6 \mathrm{~m}$, $H=1.2 \mathrm{~m}, \quad \lambda_{1}=83 \mathrm{~W} /(\mathrm{m} \cdot \mathrm{K}), \quad \lambda_{2}=54 \mathrm{~W} /(\mathrm{m} \cdot \mathrm{K}), \quad a_{1}=2.33 \cdot 10^{-5} \mathrm{~m}^{2} / \mathrm{s}^{\alpha}$, $a_{2}=1.474 \cdot 10^{-5} \mathrm{~m}^{2} / \mathrm{s}^{\alpha}, a_{\infty}=200 \mathrm{~W} /\left(\mathrm{m}^{2} \cdot \mathrm{K}\right)$. Moreover, the ambient temperature is $T_{\infty}=40^{\circ} \mathrm{C}$, the initial temperature in the inner solid cylinder and the outer concentric layer are $F_{1}=100^{\circ} \mathrm{C}$ and $F_{2}=40^{\circ} \mathrm{C}$, respectively.

In the first example, the inner heat source is specified by $g_{1}=2 \cdot 10^{5} \mathrm{~W} / \mathrm{m}^{3}$ and $g_{2}=0$ for $0 \leq z \leq H$. The non-dimensional temperature as functions of $\hat{r}=r / r_{2}$ for a different order of the fractional derivative $\alpha$ and for time $t=60 \mathrm{~s} ; 600 \mathrm{~s} ; 3600 \mathrm{~s}$; $18000 \mathrm{~s}$ is presented in Figure 2. In all cases, the temperature of the cylinder is higher for the higher order of the derivative $\alpha$ occurring in the mathematical model. As expected, the temperature increases with increasing time $t$.
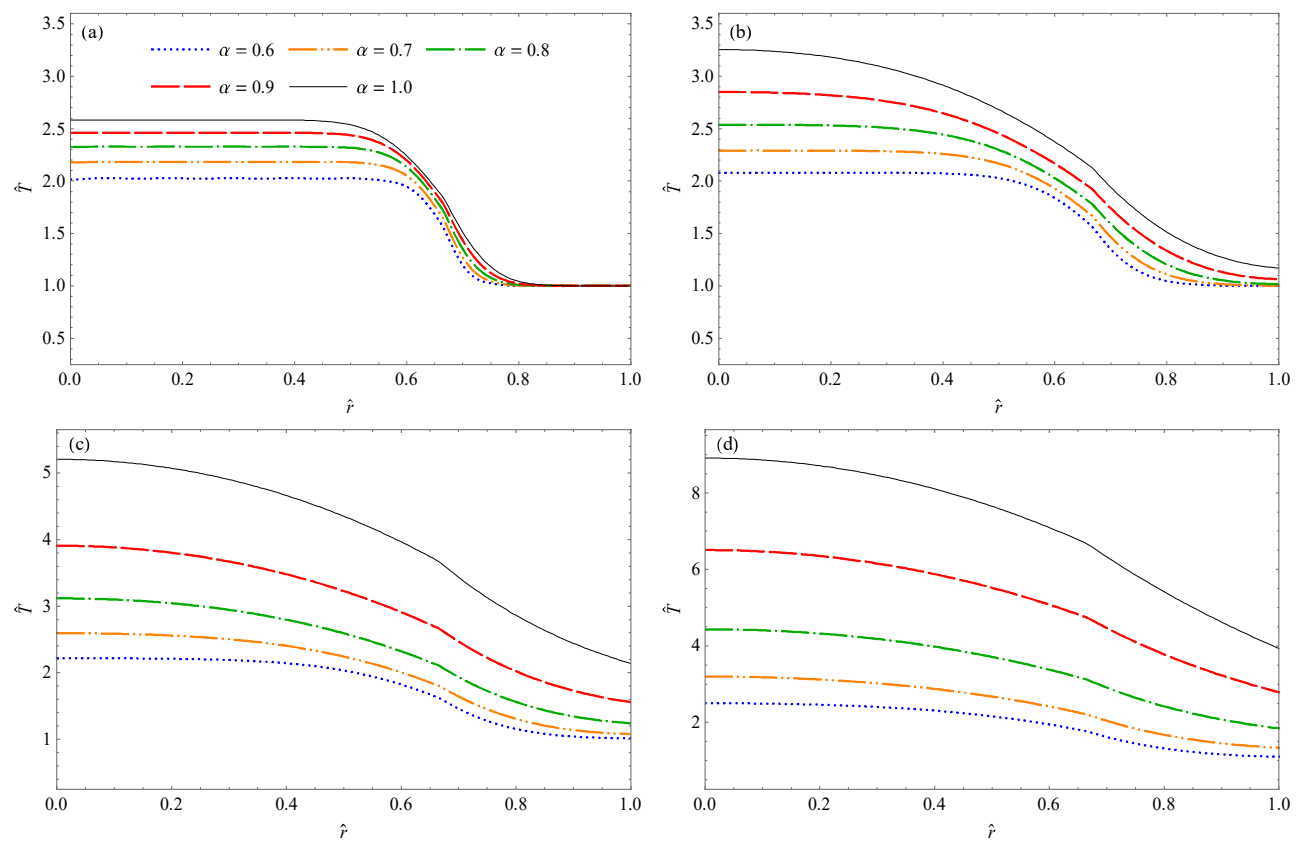

Fig. 2. Non-dimensional temperature $\hat{T}(t, \hat{r}, 0)$ as a function of the radial coordinate of $\hat{r}=r / r_{2}$ for different values of the fractional order $\alpha$ and different time $t$ :

a) $t=60 \mathrm{~s}, \mathrm{~b}) t=600 \mathrm{~s}, \mathrm{c}) t=3600 \mathrm{~s}, \mathrm{~d}) t=18000 \mathrm{~s}$

In the second example, we assume that the inner heat source is active in a part of the inner solid cylinder, i.e. the heat source is specified by the functions $g_{1}=2 \cdot 10^{5} \mathrm{~W} / \mathrm{m}^{3}$ for $0 \leq z \leq h, g_{1}=0$ for $h<z \leq H$ and $g_{2}=0$ for $0 \leq z \leq H$. The temperature as functions of $\hat{z}=z / H$ at time $t=60 \mathrm{~s} ; 600 \mathrm{~s} ; 3600 \mathrm{~s} ; 18000 \mathrm{~s}$ is shown in Figure 3. The calculations were performed for $\alpha=0.6 ; 0.7 ; 0.8 ; 0.9 ; 1.0$. 
It can be observed that the temperature of the cylinder increases with increasing time $t$ for all values of the order $\alpha$.
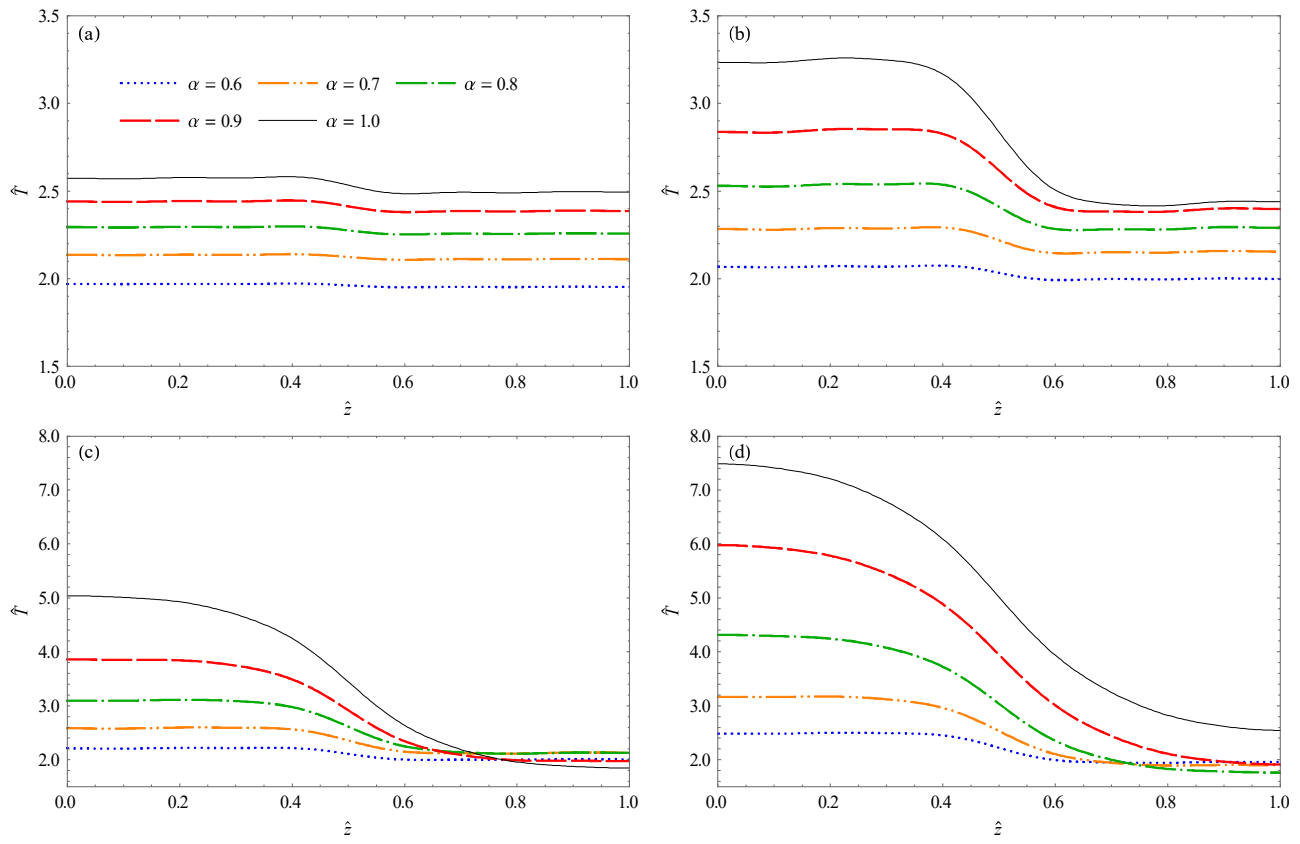

Fig. 3. Non-dimensional temperature $\hat{T}(t, 0, \hat{z})$ as a function of the $\hat{z}$-coordinate $(\hat{z}=z / H)$ for different values of the fractional order $\alpha$ and different time $t$ : a) $t=60 \mathrm{~s}, \mathrm{~b}) t=600 \mathrm{~s}, \mathrm{c}) t=3600 \mathrm{~s}, \mathrm{~d}) t=18000 \mathrm{~s}$

\section{Conclusions}

In the paper, an analytical solution of the problem of time-fractional heat conduction in a composite cylinder has been derived. In the mathematical model, the heat transfer in the direction parallel to the cylinder axis and in the radial direction was taken in the consideration. The function describing the temperature distribution in the cylinder in the form of double series of eigenfunctions is presented. Numerical examples show the effect of the Caputo time-derivative-order occurring in the heat conduction model on the temperature distribution in the cylinder. The temperature in the cylinder calculated according to the fractional heat conduction model is larger for a larger order of the time derivative.

\section{References}

[1] Machado, J.T., Kiryakova, V., \& Mainardi, F. (2011). Recent history of fractional calculus. Communications in Nonlinear Science and Numerical Simulation, 16(3), 1140-1153.

[2] Valério, D., Machado, J., \& Kiryakova, V. (2014). Some pioneers of the applications of fractional calculus. Fractional Calculus and Applied Analysis, 17(2), 552-578. 
[3] Podlubny, I. (1999). Fractional Differential Equations. San Diego: Academic Press.

[4] Diethelm, K. (2010). The Analysis of Fractional Differential Equations. Berlin/Heidelberg: Springer-Verlag.

[5] Kilbas, A.A., Srivastava, H.M., \& Trujillo, J.J. (2006). Theory and Applications of Fractional Differential Equations. Amsterdam: Elsevier.

[6] Ciesielski, M., \& Blaszczyk, T. (2018). An exact solution of the second order differential equation with the fractional/generalised boundary conditions. Advances in Mathematical Physics, $2018,7283518$.

[7] Siedlecki, J., Ciesielski, M., \& Blaszczyk, T. (2015). Transformation of the second order boundary value problem into integral form - different approaches and a numerical solution. Journal of Applied Mathematics and Computational Mechanics, 14(3), 103-108.

[8] Ozisik, M.N., \& Tzou, D.Y. (1994). On the wave theory in heat conduction. Journal of Heat Transfer, 116(3), 526-535.

[9] Tzou, D.Y. (1995). The generalized lagging response in small-scale and high-rate heating. International Journal of Heat and Mass Transfer, 38(17), 3231-3240.

[10] Povstenko, Y., \& Klekot, J. (2019). Time-fractional heat conduction in two joint half-planes. Symmetry, 11(6), 800.

[11] Povstenko, Y. (2016). Time-fractional heat conduction in a two-layer composite slab. Fractional Calculus and Applied Analysis, 19(4), 940-953.

[12] Ma, J., Sun, Y., \& Yang, J. (2018). Analytical solution of dual-phase-lag heat conduction in a finite medium subjected to a moving heat source. International Journal of Thermal Sciences, $125,34-43$.

[13] Xu, G., \& Wang, J. (2018). Analytical solution of time fractional Cattaneo heat equation for finite slab under pulse heat flux. Applied Mathematics and Mechanics, 39(10), 1465-1476.

[14] Zhang, X.-Y., \& Li, X.-F. (2019). Transient response of a functionally graded thermoelastic plate with a crack via fractional heat conduction. Theoretical and Applied Fracture Mechanics, 104, 102318.

[15] Kukla, S., \& Siedlecka, U. (2017). An analytical solution to the problem of time-fractional heat conduction in a composite sphere. Bulletin of the Polish Academy of Sciences: Technical Sciences, 65(2), 179-186.

[16] Kukla, S., \& Siedlecka, U. (2018). Fractional heat conduction in a sphere under mathematical and physical Robin conditions. Journal of Theoretical and Applied Mechanics, 56(2), 339-349.

[17] Datsko, B., Podlubny, I., \& Povstenko, Y. (2019). Time-fractional diffusion-wave equation with mass absorption in a sphere under harmonic impact. Mathematics, 7(5), 433.

[18] Ning, T.-H., \& Jiang, X.-Y. (2011). Analytical solution for the time-fractional heat conduction equation in spherical coordinate system by the method of variable separation. Acta Mechanica Sinica, 27, 994-1000.

[19] Yu, B., \& Jiang, X. (2019). Temperature prediction by a fractional heat conduction model for the bi-layered spherical tissue in the hyperthermia experiment. International Journal of Thermal Sciences, 145, 105990.

[20] Jiang, X., \& Xu, M. (2010). The time fractional heat conduction equation in the general orthogonal curvilinear coordinate and the cylindrical coordinate systems. Physica A, 389, 3368-3374.

[21] Povstenko, Y. (2012). Time-fractional radial heat conduction in a cylinder and associated thermal stresses. Archive of Applied Mechanics, 82, 345-362.

[22] Povstenko, Y. (2014). Axisymmetric solution to time-fractional heat conduction equation in an infinite cylinder under local heating and associated thermal stresses. International Journal of Mechanics, 8(1), 383-390.

[23] Ezzat, M.A., \& El-Bary, A.A. (2016). Effects of variable thermal conductivity and fractional order of heat transfer on a perfect conducting infinitely long hollow cylinder. International Journal of Thermal Sciences, 108, 62-69.

[24] Blasiak, S. (2018). Time-fractional Fourier law in a finite hollow cylinder under Gaussiandistributed heat flux. EPJ Web of Conferences, 180, 02008. 